\title{
Comprehensive metabolic profiling of Parkinson's disease by liquid chromatography-mass spectrometry
}

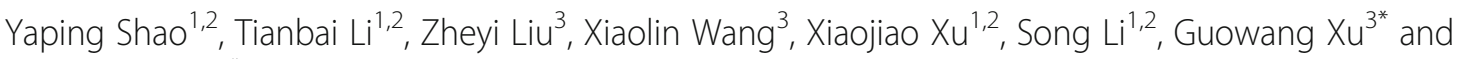
Weidong $L e^{1,2,4^{*}}$ (iD

\begin{abstract}
Background: Parkinson's disease (PD) is a prevalent neurological disease in the elderly with increasing morbidity and mortality. Despite enormous efforts, rapid and accurate diagnosis of PD is still compromised. Metabolomics defines the final readout of genome-environment interactions through the analysis of the entire metabolic profile in biological matrices. Recently, unbiased metabolic profiling of human sample has been initiated to identify novel PD metabolic biomarkers and dysfunctional metabolic pathways, however, it remains a challenge to define reliable biomarker(s) for clinical use.
\end{abstract}

Methods: We presented a comprehensive metabolic evaluation for identifying crucial metabolic disturbances in PD using liquid chromatography-high resolution mass spectrometry-based metabolomics approach. Plasma samples from 3 independent cohorts ( $n=460,223$ PD, 169 healthy controls (HCs) and 68 PD-unrelated neurological disease controls) were collected for the characterization of metabolic changes resulted from PD, antiparkinsonian treatment and potential interferences of other diseases. Unbiased multivariate and univariate analyses were performed to determine the most promising metabolic signatures from all metabolomic datasets. Multiple linear regressions were applied to investigate the associations of metabolites with age, duration time and stage of PD. The combinational biomarker model established by binary logistic regression analysis was validated by 3 cohorts.

Results: A list of metabolites including amino acids, acylcarnitines, organic acids, steroids, amides, and lipids from human plasma of 3 cohorts were identified. Compared with HC, we observed significant reductions of fatty acids (FFAs) and caffeine metabolites, elevations of bile acids and microbiota-derived deleterious metabolites, and alterations in steroid hormones in drug-naïve PD. Additionally, we found that L-dopa treatment could affect plasma metabolome involved in phenylalanine and tyrosine metabolism and alleviate the elevations of bile acids in PD. Finally, a metabolite panel of 4 biomarker candidates, including FFA 10:0, FFA 12:0, indolelactic acid and phenylacetyl-glutamine was identified based on comprehensive discovery and validation workflow. This panel showed favorable discriminating power for PD.

(Continued on next page)

\footnotetext{
*Correspondence: xugw@dicp.ac.cn; wdle_sibs@163.com

${ }^{3}$ CAS Key Laboratory of Separation Science for Analytical Chemistry, Dalian Institute of Chemical Physics, Chinese Academy of Sciences, 457 Zhongshan Road, Dalian 116023, China

${ }^{1}$ Center for Clinical Research on Neurological Diseases, The First Affiliated Hospital, Dalian Medical University, 193 Lianhe Road, Dalian, China Full list of author information is available at the end of the article
}

(c) The Author(s). 2021 Open Access This article is licensed under a Creative Commons Attribution 4.0 International License, which permits use, sharing, adaptation, distribution and reproduction in any medium or format, as long as you give appropriate credit to the original author(s) and the source, provide a link to the Creative Commons licence, and indicate if changes were made. The images or other third party material in this article are included in the article's Creative Commons licence, unless indicated otherwise in a credit line to the material. If material is not included in the article's Creative Commons licence and your intended use is not permitted by statutory regulation or exceeds the permitted use, you will need to obtain permission directly from the copyright holder. To view a copy of this licence, visit http://creativecommons.org/licenses/by/4.0/ The Creative Commons Public Domain Dedication waiver (http://creativecommons.org/publicdomain/zero/1.0/) applies to the data made available in this article, unless otherwise stated in a credit line to the data. 
(Continued from previous page)

Conclusions: This study may help improve our understanding of PD etiopathogenesis and facilitate target screening for therapeutic intervention. The metabolite panel identified in this study may provide novel approach for the clinical diagnosis of PD in the future.

Keywords: Parkinson's disease, Metabolomics, Biomarker, Metabolic disturbances, Bile acid profile

\section{Background}

Parkinson's disease (PD) is the most prevailing movement disorder and represents the second most common neurodegenerative disease, affecting approximately $1 \%$ of the population above 60 years $[1,2]$. The main neuropathological characteristics of PD are marked loss of dopaminergic neurons within substantia nigra and the presence of intracytoplasmic $\alpha$-synuclein-containing Lewy bodies, manifesting as reduced facilitation of voluntary movement $[2,3]$. The current diagnosis of PD essentially relies on evaluation of clinical signs. Although neuroimaging technologies have improved the diagnosis and staging of PD, these detections are expensive and labor intensive $[4,5]$. Therefore, many studies have been dedicated to the discovery of biomarkers that may assist the diagnosis of PD. However, no peripheral blood derived biomarkers have been used clinically at present [6-8]. Accumulated evidence indicated that PD is multifactorial; a combination of age, genetics and environmental factors might contribute to its onset and progression $[9,10]$.

Emerging evidence indicated that peripheral alterations including metabolic dysregulations might precede and contribute to neurodegeneration [11-14]. Deciphering the molecular networks that distinguish PD from healthy individuals and patients with other PD-unrelated diseases might lead to novel insight into PD pathogenesis and the identification of crucial biomarkers. Liquid chromatography-mass spectrometry- (LC-MS) based metabolomics is a powerful tool to profile metabolite changes. It has been utilized to decipher metabolic reprogramming in many types of disease including neurodegenerative disorders $[12,15,16]$. Previous studies indicated the involvement of oxidative stress and dyshomeostasis in metabolism of catecholamine, tryptophan and caffeine in PD [3, 16, 17]. Several potential biomarkers have been identified, such as acylcarnitines [18], quinolinic acid (QA)/kynurenic acid (KA) ratio [19], N8-acetyl spermidine and lipids [10, 20]. However, many studies cannot corroborate each other, possibly due to the limited sample size, lack of validation cohort and confounding factors from clinical heterogeneity, analytical methodology and antiparkinsonian medication. Recently, bile acid (BA) metabolism has been linked to liver diseases, diabetes, inflammatory bowel diseases and neurodegenerative disorders [12, 21, 22]. In addition to their important roles in lipid digestion and absorption, BAs act as signaling molecules by activating membrane and nuclear receptors as well as ion channels [23].
However, to the best of our knowledge, there has been no systematic study on the profiling of BAs in PD population.

Herein, we utilized a LC-MS based untargeted metabolomics approach to investigate the metabolic changes associated with PD in 3 well-characterized cohorts. Using a method of targeted extraction and integration of the chromatographic peak, we also presented a comprehensive analysis of BA profiles in PD. Additionally, the influences from various variables (gender, age, duration, stage, and pharmacological treatment) to the level of metabolites were also investigated. We aimed to identify the most promising metabolic biomarkers for the diagnosis of PD, and corresponding metabolic pathways that might contribute to a better understanding of the biochemical impairments involved in the disease.

\section{Methods \\ Participants}

Totally, 460 plasma samples including 223 from PD, 169 from healthy controls (HCs) and 68 from patients with PD-unrelated neurological diseases were enrolled at the First Affiliated Hospital of Dalian Medical University. PD patients were diagnosed by at least two experienced neurologists based on the Movement Disorder Society Clinical Diagnostic Criteria for Parkinson's disease, and their favorite response to L-dopa therapy [24]. HC subjects were recruited from the Health Examination Center. In cohort 1 , all the PD patients were drug-naïve. In cohort 2, 97 individuals were included, of which 51 were treated and 14 were drug-naïve, and 32 were $\mathrm{HC}$. In cohort 3, apart from PD and HC, a matched PD-unrelated neurological disease control (NDC) group comprised of 27 cerebrovascular diseases, 9 epilepsy, 9 peripheral vertigo, 8 peripheral neuropathy, 8 anxiety/sleep disorders, 5 syncope and 2 myasthenia gravis were included. Most of the patients in NDC did not receive regular medications, except for epilepsy patients who were routinely treated with antiepileptic drugs. Detailed descriptions of the participants are given in Table 1. All subjects or their legally authorized caregivers provided informed consents and this study was approved by the Ethics Committee of the hospital.

\section{Biospecimen collection and processing}

Fasting venous blood samples were collected into ethylene diamine tetra-acetic acid containing 
Table 1 Study population features

\begin{tabular}{|c|c|c|c|c|}
\hline & $\mathrm{PD}(n=223)$ & $\mathrm{HC}(n=169)$ & NDC $(n=68)$ & $p$ value $^{a}$ \\
\hline \multicolumn{5}{|l|}{ Cohort 1} \\
\hline Number of individuals & 36 & 43 & - & \\
\hline Age, mean \pm SE & $64.4 \pm 1.5$ & $65.5 \pm 1.2$ & - & 0.6826 \\
\hline Gender (m/f) & $20 / 16$ & $25 / 18$ & - & 0.8173 \\
\hline Duration of disease (year), mean $\pm \mathrm{SE}$ & $4.4 \pm 0.8$ & - & - & \\
\hline $\mathrm{H} \& \mathrm{Y}$ stage, mean $\pm \mathrm{SE}$ & $2.1 \pm 0.1$ & - & - & \\
\hline \multicolumn{5}{|l|}{ Cohort 2} \\
\hline Number of individuals & 65 & 32 & - & \\
\hline Age, mean $\pm S E$ & $66.2 \pm 1.3$ & $64.6 \pm 1.7$ & - & 0.6367 \\
\hline Gender (m/f) & $36 / 29$ & $18 / 14$ & - & 0.9651 \\
\hline Duration of disease (year), mean $\pm \mathrm{SE}$ & $5.1 \pm 0.5$ & - & - & \\
\hline $\mathrm{H} \& Y$ stage, mean $\pm \mathrm{SE}$ & $2.1 \pm 0.1$ & - & - & \\
\hline \multicolumn{5}{|l|}{ Cohort 3} \\
\hline Number of individuals & 122 & 94 & 68 & \\
\hline Age, mean \pm SE & $68.2 \pm 1.0$ & $68.6 \pm 0.8$ & $68.6 \pm 1.1$ & 0.9576 \\
\hline Gender (m/f) & $68 / 54$ & $51 / 43$ & $37 / 31$ & 0.9720 \\
\hline Duration of disease (year), mean $\pm \mathrm{SE}$ & $5.6 \pm 0.4$ & - & - & \\
\hline $\mathrm{H} \& \mathrm{Y}$ stage, mean $\pm \mathrm{SE}$ & $2.4 \pm 0.1$ & - & - & \\
\hline
\end{tabular}

${ }^{a}$ The Mann-Whitney $\mathrm{U}$ test (PD and $\mathrm{HC}$ ) or one-way ANOVA (PD, HC and NDC) was used to calculate the statistical significance difference in age distribution between the groups in each cohort. A chi-square test was applied to investigate the difference in gender composition

In cohort1, all the PD patients were drug-naïve patients. In cohort 2, 14 were drug-naïve, 51 were treated PD patients (L-dopa-treated, pramipexol-treated or the combination of L-dopa and pramipexol-treated). In cohort 3, 27 were drug-naïve, 95 were treated PD patients. Most of the patients in NDC group did not receive regular medications, except for 9 patients with epilepsy who were treated with antiepileptic drugs. There is no significant difference in caffeine consumption between PD and controls. $\mathrm{m} / \mathrm{f}$ indicates ratio of the number of males to the number of females

vacutainers (Insepack, SEKISUI medical technology) by direct venipuncture. Subsequently, plasma samples were transferred into sterile tubes after centrifugation at $3000 \mathrm{rpm}$ for $5 \mathrm{~min}$ and stored at $-80^{\circ} \mathrm{C}$. Before metabolomics analysis, plasma samples were visually checked for hemolysis, and there were no hemolytic specimens used in our study. Plasma samples were prepared as previously described with slight modifications [25]. $130 \mu \mathrm{L}$ of plasma was deproteinized with 4 volumes of methanol containing internal standards (ISs, Table S1). After centrifugation at $13,000 \mathrm{~g}$ for $10 \mathrm{~min}$, the resulting supernatant was divided into two aliquots and lyophilized. The dried samples were reconstituted in $65 \mu \mathrm{L}$ of methanol/water $(1 / 3)$ and analyzed by LC-MS operated in ESI positive (ESI+, basic species) and negative (ESI-, acidic species) modes. To evaluate the repeatability of sample pretreatment and monitor the stability of instrument analysis, quality control (QC) samples were made by mixing equal amounts of each sample, prepared identically to the analytical sample, and analyzed after 10 sample runs. Additionally, blank samples with ultrapure water instead of plasma were made and treated with the same method and analyzed before the sequence was run to assess potential background interference during the experimental process.

\section{LC-MS analysis}

Metabolic profiling was performed on an Ultra Performance Liquid Chromatography (UPLC, Waters, Manchester, UK) coupled with tripleTOF 5600 plus (Applied Biosystems, Foster City, CA) MS system. In ESI+ mode, extracts were retained and gradient eluted from an ACQUITY UPLC BEH C8 column using water and acetonitrile with $0.1 \%$ formic acid solution. In ESImode, extracts were retained and gradient eluted from an ACQUITY UPLC HSS T3 column using water and 95\% methanol containing $6.5 \mathrm{mM}$ ammonium bicarbonate. Detailed chromatographic and MS conditions are given in Supplementary Materials. Instrument control and data acquisition were conducted using Analyst TF 1.7 software.

\section{Raw data preprocessing}

Total ion chromatograms were analyzed using Peakview (version 1.2.0.3, Applied Biosystems). The acquired raw data were imported into Marker View (version 1.2.1.1, Applied Biosystems) for peak extraction and alignment. 
Features that detected in at least $80 \%$ of acquired samples in disease or control group were retained [26]. Besides, due to the automatic peak alignment process, the information of low abundance of metabolites may be lost. To obtain a comprehensive and accurate profile of BA, we performed a method of targeted extraction and integration of the chromatographic peak using MultiQuant (version 2.1, Applied Biosystems) software (Supplementary Materials). Prior to statistical analysis, original datasets were calibrated by ISs. Each ion feature in QC sample was calibrated with all ISs and the relative standard deviation (RSD) after each calibration was calculated. We used IS that can achieve the minimum RSD in QC sample to calibrate the ion features in analytical samples. For metabolites identified in 3 datasets, the original peak areas were calibrated using identical ISs.

\section{Metabolite identification}

The processing of metabolite identification was carried out with the OSI/SMMS software [27]. In brief, the retention time of each ion feature was calibrated using ISs to estimate fluctuations between batches. The calibrated retention time and MS data of ion features in analyzed samples were searched against an in-house database by comparing the qualitative information of each metabolite with that of reference chemical standards. Comprehensive procedures including precursor ion alignment and ion fusion, database searching and scoring were applied to remove the artifacts and background noise [27].

\section{Statistics}

SIMCA (version 13.0.0.0., Umetrics AB, Umea, Sweden) was used to perform multivariate analysis including principal component analysis (PCA), partial least square discriminant analysis (PLS-DA) and orthogonal PLS-DA (OPLS-DA). Permutation test was conducted to avoid a potential risk of overfitting. We used the Mann-Whitney $\mathrm{U}$ test to calculate the statistical significance. A standard Benjamini-Hochberg method was applied to control the false discovery rate (FDR) for multiple hypothesis testing [28]. Linear regressions were applied to investigate associations of metabolites with age, duration and disease severity using PASW Statistics (version 18.0.0). Hierarchical cluster analysis was performed using the $\mathrm{MeV}$ software package (version 4.8.1). Binary logistic regression analysis was applied to generate a mathematical model for PD discrimination. The predictive performance of the model was assessed by estimating the area under the receiver operating characteristic (ROC) curve (AUC), which is commonly used to evaluate overall discriminant ability [29].

\section{Results}

The demographic and clinical characteristics of the involved subjects are presented in Table 1. A total of 460 individuals were enrolled and divided into 3 independent cohorts (Fig. 1). There was no significant difference in age distribution or gender composition between the groups in each cohort, which indicated that the subjects in each group were comparable.

After instrumental analysis, peak detection and alignment, and metabolite recognition, 226, 202 and 204 metabolites were identified in cohort 1 , cohort 2 and cohort 3 , respectively (Fig. 2). The data matrices of the identified metabolites were used for the following statistical analysis. We first assessed the metabolomic data quality of the datasets drawn from 3 independent cohorts. As shown in Figure S1a-1c, the QC samples clustered in the center on the PCA score plots, suggesting that the analyses were repeatable and robust. Then we further analyzed the RSD of metabolites in QC samples (Figure S1d-1f), 90.3, 99.5 and $98.5 \%$ of the metabolites had RSD values $<30 \%$ in each cohort, which further confirmed the reliability of the data.

Considering that the detections of fatty acids (FFAs) might be interfered with residuals from plastic containers [30], we further analyzed and compared the contents of FFAs in blank (ultrapure water) and analytical samples (QC samples). Totally, 35 FFAs were detected in plasma samples. Among them, 8 FFAs were not detectable in blank sample. For the rest of 27 FFAs, the contents of 19 FFAs in the blank were less than $10 \%$ of the analytical sample. We found that the contents of only 2 FFAs were more than $30 \%$ of the analytical sample (Table S2). Besides, we used stable isotope labeled FFAs as IS to calibrate the original raw data to reduce systematic errors drawn from sample pretreatment and instrumental analysis processes. After IS calibration, 94\% FFAs had RSDs of less than 15\% and all the FFAs had RSDs of less than 30\% in QC samples (Table S2), which implied that the interference derived from plastic containers did not significantly affect the detection reproducibility and relative quantification of FFA in this study.

\section{Metabolic signatures of drug-naïve PD}

A comparative study involving drug-naïve PD (DN-PD) and matched $\mathrm{HC}$ was initially performed to investigate the metabolic signatures of PD and rule out potentially confounding effects of symptomatic medications. The metabolic differences between males and females in DN$\mathrm{PD}$ and $\mathrm{HC}$ were firstly investigated. As shown in Figure $\mathbf{S 2}$, there was no clear separation between males and females in both HC and DN-PD on the PCA score plot. By performing univariate analysis with FDR calibration, 24 metabolites including acetylcholine, creatinine, several amino acids, phosphatidylcholines (PCs) and sphingomyelins (SMs) were found different between genders in HC (Table S3). However, no gender difference was found in DN-PD. These findings suggested that the 


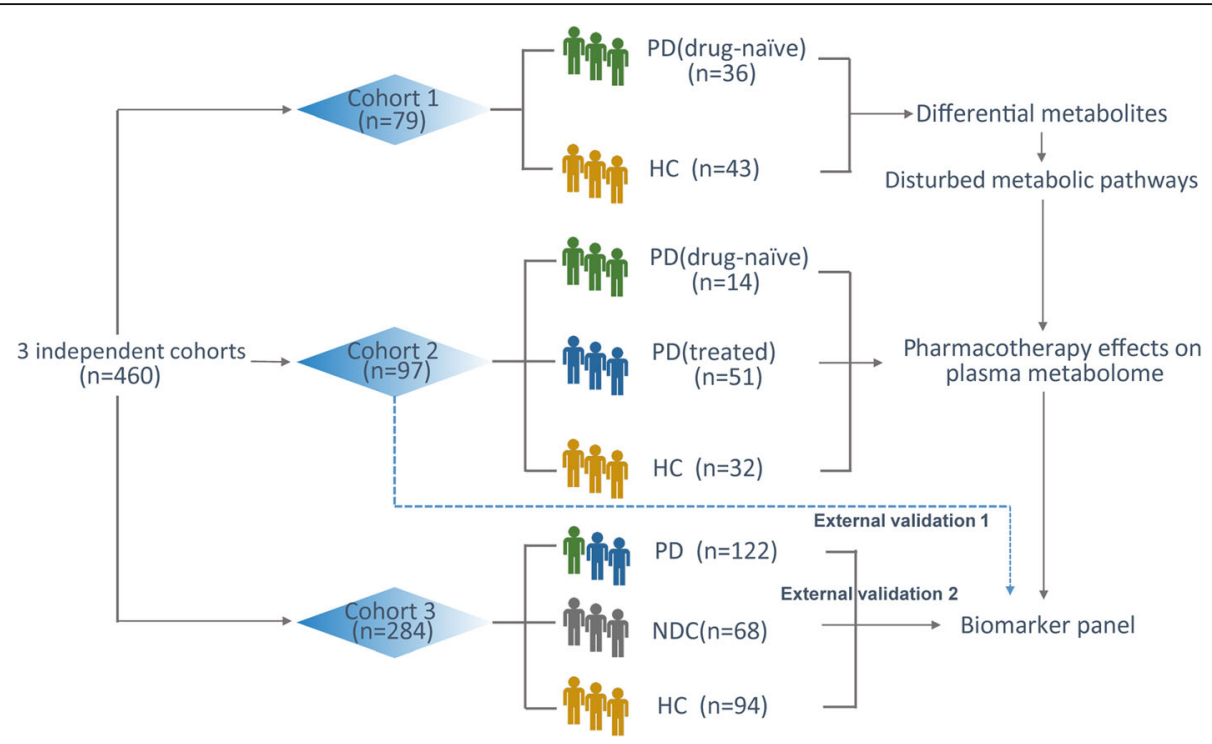

Fig. 1 Flow chart of the experimental design. Totally, 460 plasma samples were collected and divided into three independent data sets for metabolomics analysis

metabolic profile of PD was significantly altered compared to that of healthy individuals.

To maximize identification of differential metabolites in PD patients, we constructed a PLS-DA model. The plasma metabolome of DN-PD was clearly separated from $\mathrm{HC}$ on the score plot (Fig. 3a). Permutation test was performed to ensure that the model was not overfitted (Figure S3a). Of the 226 metabolites, 75 metabolites contributed significantly to the distinction of DN-PD and HC with VIP values $>1$. We further performed a Mann-Whitney $U$ test with FDR calibration and found 60 metabolites were significantly changed (Fig. 3b). The overlapped 50 metabolites were considered as differential metabolites in PD (Fig. 3c). Of these, levels of 43 metabolites were decreased, including acyl carnitines, PCs, FFAs, FFA amides (FFADs), indolelactic acid, trigonelline and kynurenine, among others. Conversely, levels of 7 metabolites including phenylacetyl-L-glutamine, $p$-cresol glucuronide, $p$-cresol sulfate, proline, cortisol, corticosterone, and phosphate were significantly increased in PD (Fig. 3d, Table S4). Based on these differential metabolites identified in PD, we further carried out pathway analysis and found that metabolic dysregulations in unsaturated FFAs biosynthesis (especially linoleic acid, linolenic acid, and arachidonic acid metabolism), steroid hormone biosynthesis, pantothenate and CoA biosynthesis and amino acids metabolism might be involved in PD etiopathogenesis (Figure S4).

Then, we investigated the associations of the differential metabolites with disease severity, duration, and age.

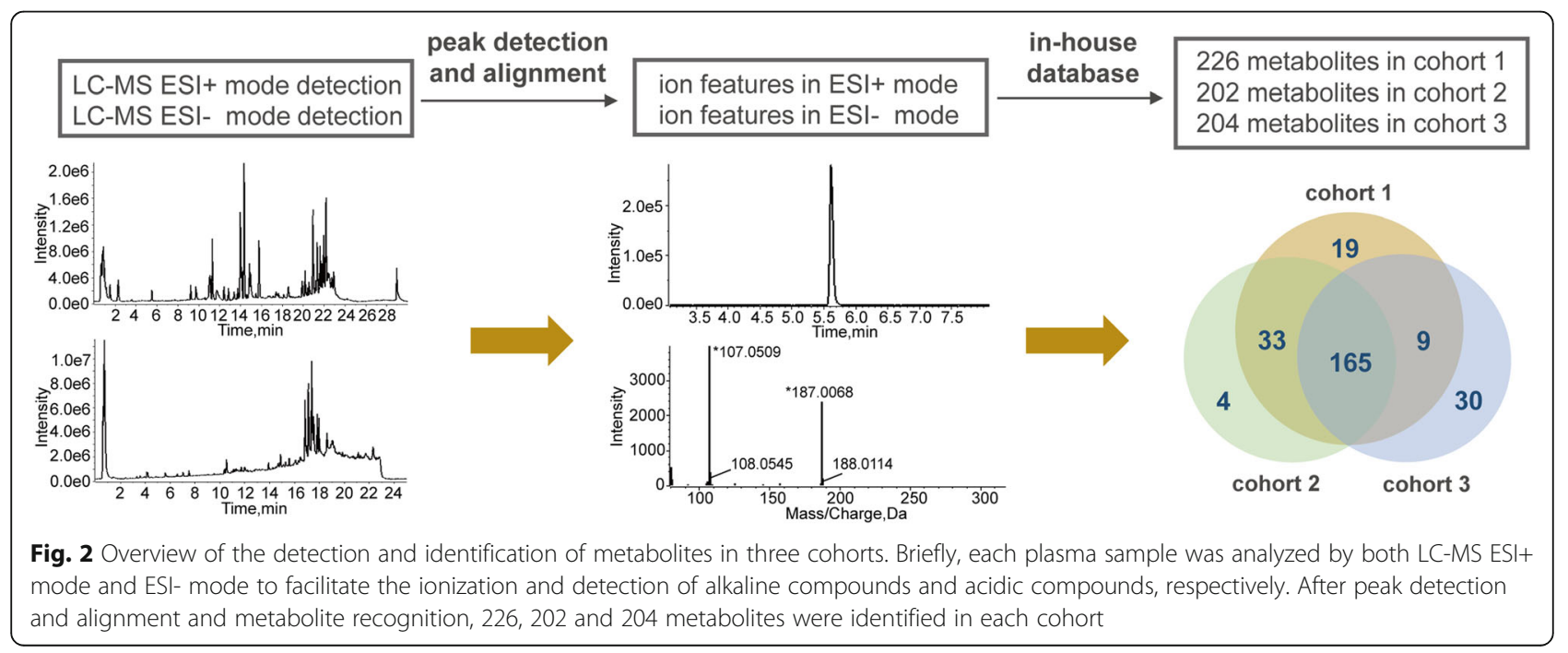



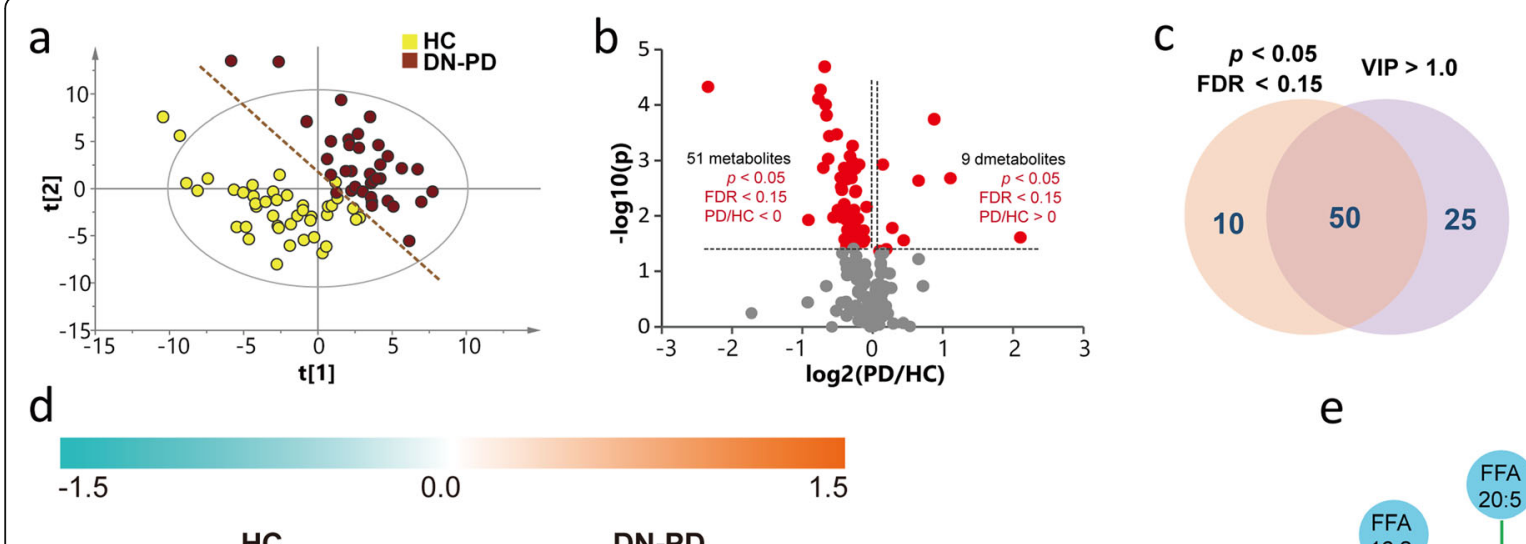

\section{e}
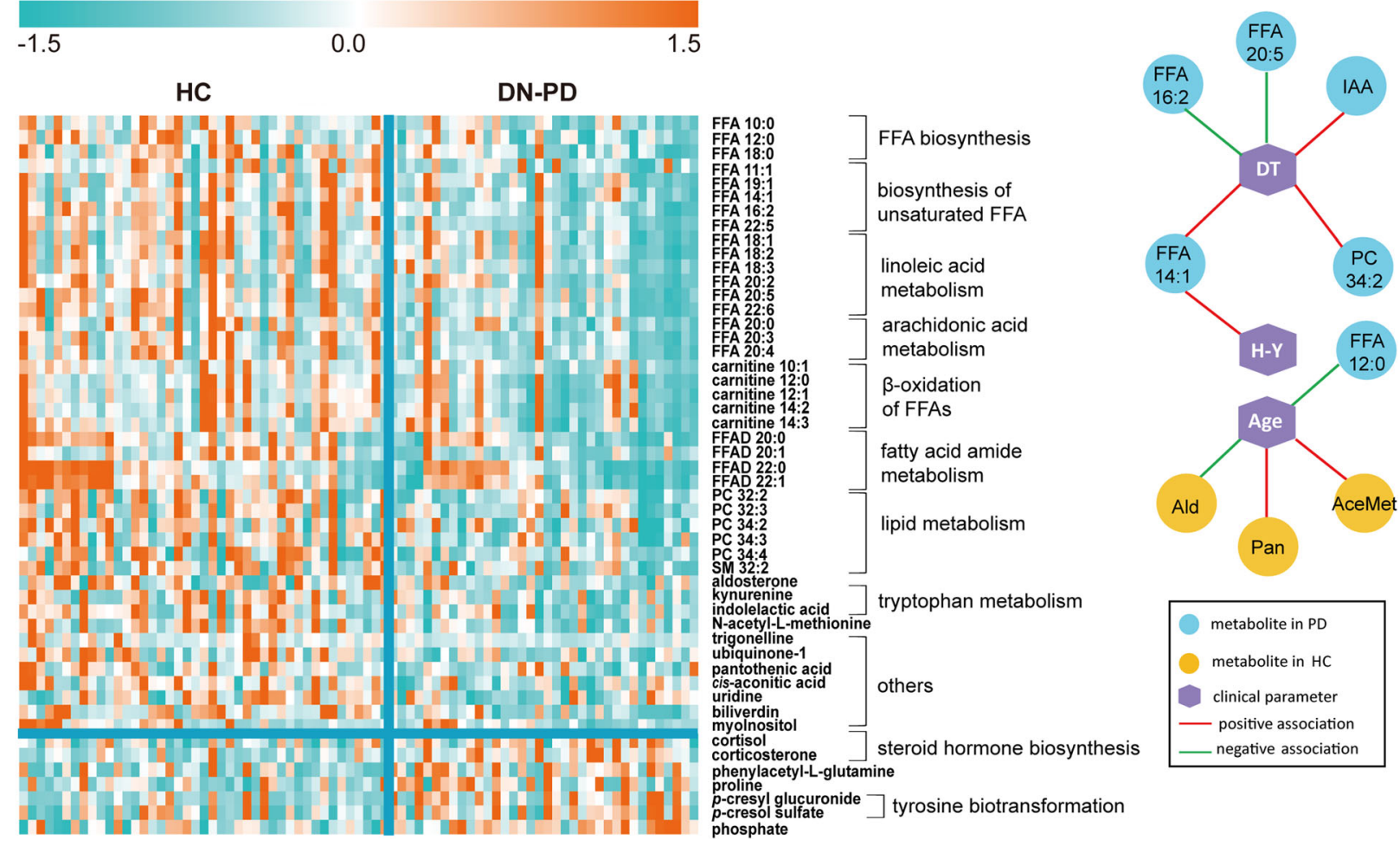

Fig. 3 Altered metabolic profiles in drug-naïve PD compared with HC. a PLS-DA score plot of DN-PD and HC in cohort 1. R2X $=0.212, R 2 Y=0.758, Q 2=$ 0.594. The analysis of variance was based on cross-validated prediction residuals (CV-ANOVA) data: $p=9.6139 \mathrm{E}-014, \mathrm{~F}$ factor $=26.7611$. $\mathbf{b}$ Volcano plot of the differential metabolites in DN-PD filtered by univariate analysis. $\mathbf{c}$ Venn plot of the differential metabolites filtered by PLS-DA model and univariate analysis. $\mathbf{d}$ Heat map of the 50 differential metabolites in DN-PD. Blue indicates a decreased level, orange indicates an increased level. e Associations of metabolites with disease severity, duration time and age. DT: duration time; IAA: indolelactic acid; Ald: aldosterone; Pan: pantothenic acid;

AceMet: N-acetyl-L-methionine

The disease severity of PD was assessed based on Modified Hoehn - Yahr (H-Y) staging [31]. We found that FFA 14:1 was positively associated with disease severity (Fig. 3e, Table S5). Levels of FFA 14:1, PC 34:2 and indolelactic acid were positively associated with the duration time of the disease, whereas FFA 20:5 and FFA 16: 2 were negatively associated (Fig. 3e, Table S6). Interestingly, metabolites in $\mathrm{HC}$ and $\mathrm{PD}$ showed different age associations. Aldosterone, pantothenic acid, and $\mathrm{N}$ acetyl-L-methionine were associated with age in $\mathrm{HC}$, however, only 1 metabolite FFA 12:0 showed association with age in PD (Fig. 3e, Table S7). These findings provided further evidence that $\mathrm{HC}$ and PD had different metabolic patterns.

\section{Impacts of drug therapies on plasma metabolome of PD} Generally, PD patients are usually treated with different types of antiparkinsonian medication. To assess the possible drug-induced changes in plasma metabolome, we recruited an independent population including DN-PD, treated PD (L-dopa-treated PD (DO-PD), pramipexoletreated PD (PR-PD) and the combination of L-dopa and pramipexole-treated PD (CO-PD)) and $\mathrm{HC}$ for metabolomics analysis. 
As shown in Figure S1b, the plasma metabolome of PD showed a trend of separation from HC, whereas different subgroups of PD displayed partial overlap. To investigate the impacts of medications on plasma metabolome, we constructed PLS-DA models between DN-PD and different types of treated PD. We found that the metabolome of DN-PD showed a difference from that of DO-PD on the score plot of PLS-DA model without overfitting (Fig. 4a, Figure S3b). However, PLS-DA models between DN-PD and PR-PD or CO-PD did not show clear separation. These findings implied that Ldopa treatment could cause significant impact on the plasma metabolome. However, neither pramipexole treatment nor the combination of L-dopa and pramipexole treatment could cause significant impacts. Based on multivariate and univariate statistical analyses (VIP value, $p$ value and FDR), 11 metabolites including phenol sulphate, lysophosphatidylcholine (LPC), PC, SM, L-3methoxytrosine, phenylalanine, etc. were found relevant to L-dopa treatment. We found that L-dopa treatment resulted in a remarkable increase in the level of L-3methoxytyrosine, which is a major metabolite of L-dopa (Fig. 4b). Based on univariate statistical analysis solely, no metabolites were found relevant to pramipexole treatment alone, and 3 metabolites including phenylalanine, L-3-methoxytyrosine and PE o-38:6 were related to combinational treatment (Fig. 4c).

\section{BA profiles in PD patients}

We demonstrated a significant elevation of liver-derived primary bile acid $\mathrm{CA}$, and bacterially generated secondary bile acid DCA as well as conjugated bile acid TDCA, GDCA, GDCS, TCAS and GLCAS in PD compared to HC. Notably, both L-dopa and combinational treatments could alleviate the elevations of BAs in PD patients. However, no significant alterations were found after pramipexole treatment alone (Table 2). Interestingly, UDCA and TUDCA, which were reported neuroprotective in PD [32], were decreased in the plasma of PD patients, although there was no statistical significance. By calculating the ratios of BAs, we assessed the activities of enzymes involved in BA synthesis and found a significant elevation of CA/CDCA in PD, indicating a shift in initial cholesterol metabolism from alternative pathway to primary pathway. Besides, most of the ratios were increased in DN-PD compared to $\mathrm{HC}$, the elevations of these $\mathrm{BA}$ ratios tend to be alleviated by medication.

\section{Discriminant model establishment and validation}

To further validate the metabolic changes in PD and identify potential biomarkers, we collected another set of plasma sample for metabolomics analysis. In contrast to the majority of "case-control" studies, we included not only a $\mathrm{HC}$ but also a disease control group. Because the disease control group contains a variety of disease types,

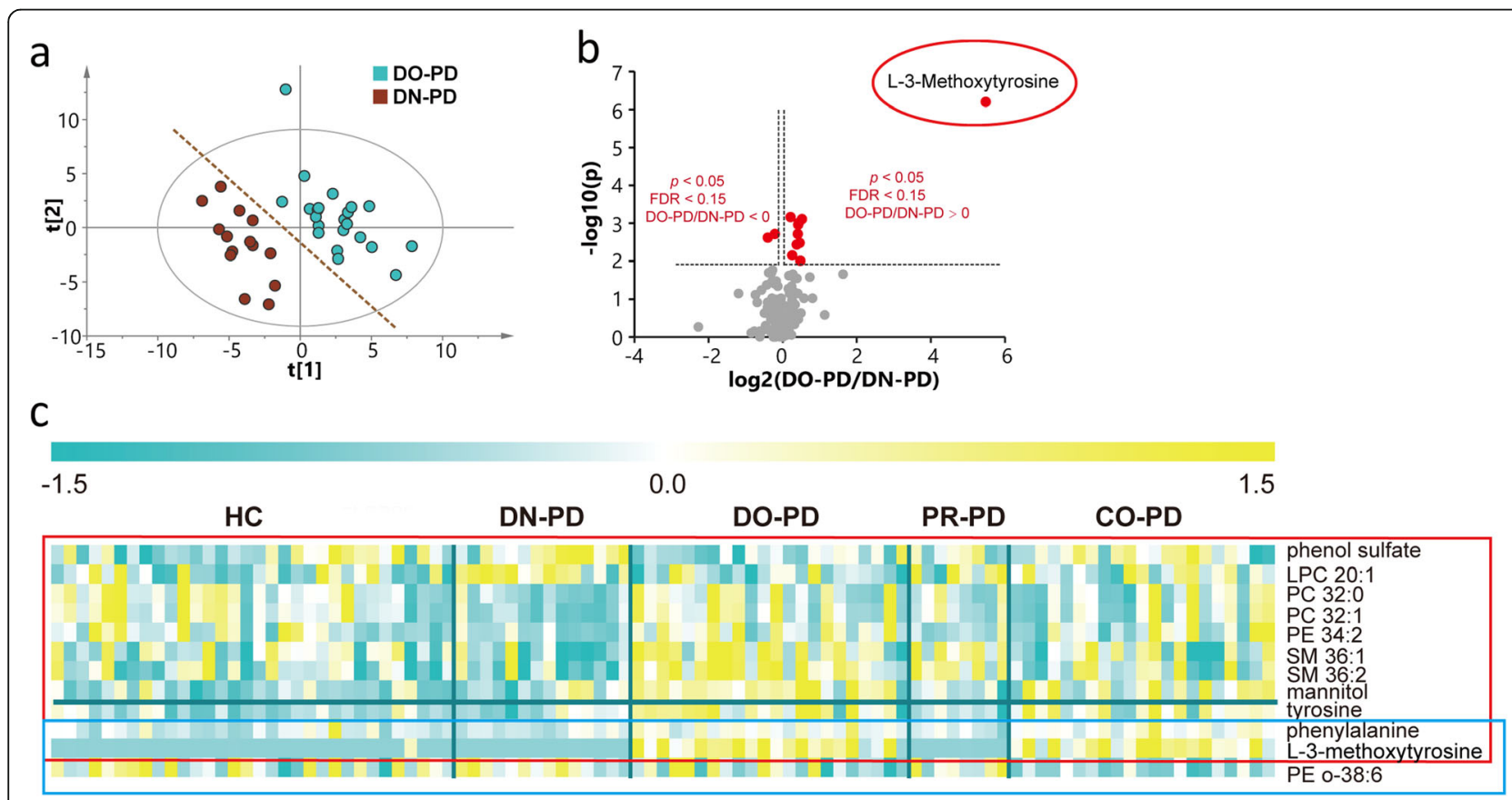

Fig. 4 Impacts of drug therapies on plasma metabolome of PD. a PLS-DA score plot of DN-PD and DO-PD in cohort 2. R2X $=0.179, R 2 Y=0.863$, $\mathrm{Q} 2=0.323$. CV-ANOVA data: $p=0.0030, \mathrm{~F}$ factor $=5.0488$. $\mathbf{b}$ Volcano plot of the significantly changed metabolites in the plasma of PD patients after L-dopa treatment. $\mathbf{c}$ Heat map of differential metabolites between treated PD and DN-PD, which showed possible drug effects to the plasma metabolome 
Table 2 Statistical results of bile acids contents and ratios in drug-naïve PD, treated PD and healthy controls in cohort 1 and cohort

\begin{tabular}{|c|c|c|c|c|c|c|c|c|c|c|c|c|c|}
\hline \multirow[t]{3}{*}{ Category } & \multirow[t]{3}{*}{ BAs/ratios } & \multirow{2}{*}{\multicolumn{3}{|c|}{$\begin{array}{l}\text { Cohort } 1 \\
\text { DN-PD vs. HC }\end{array}$}} & \multicolumn{9}{|c|}{ Cohort 2} \\
\hline & & & & & \multicolumn{3}{|c|}{ DN-PD vs. HC } & \multicolumn{3}{|c|}{ DO-PD vs. DN-PD } & \multicolumn{3}{|c|}{ CO-PD vs. DN-PD } \\
\hline & & $p$ & FDR & $\mathrm{FC}_{1}$ & $p$ & FDR & $\mathrm{FC}_{2}$ & $p$ & FDR & $\mathrm{FC}_{3}$ & $p$ & FDR & $\mathrm{FC}_{4}$ \\
\hline \multirow[t]{2}{*}{ Primary } & CA & 0.0183 & 0.1034 & 2.64 & 0.4102 & 0.6766 & 1.98 & 0.9096 & 0.9096 & 0.83 & 0.9329 & 0.9912 & 1.09 \\
\hline & CDCA & 0.1978 & 0.3422 & 1.53 & 0.4378 & 0.6766 & 1.48 & 0.5702 & 0.7455 & 0.77 & 0.8531 & 0.9912 & 0.9 \\
\hline \multirow[t]{3}{*}{ Secondary } & DCA & 0.0033 & 0.0499 & 1.76 & 0.7114 & 0.7802 & 1.02 & 0.2237 & 0.6337 & 0.81 & 0.3545 & 0.7532 & 0.90 \\
\hline & UDCA & 0.2595 & 0.4201 & 0.67 & 0.9905 & 0.9905 & 0.92 & 0.0770 & 0.2908 & 0.43 & 0.6494 & 0.9912 & 0.9 \\
\hline & LCA & 0.4026 & 0.6029 & 1.19 & 0.7837 & 0.8074 & 1.03 & 0.3384 & 0.6907 & 0.67 & 0.9866 & 1.0000 & 0.8 \\
\hline \multirow[t]{6}{*}{ Primary conjugated } & GCA & 0.0424 & 0.1601 & 1.43 & 0.0272 & 0.2314 & 1.72 & 0.0055 & 0.0376 & 0.40 & 0.0529 & 0.3594 & 0.81 \\
\hline & TCA & 0.1776 & 0.3422 & 1.31 & 0.0226 & 0.2314 & 1.99 & 0.0017 & 0.0197 & 0.29 & 0.0090 & 0.1872 & 0.7 \\
\hline & TCAS & 0.0073 & 0.0499 & 2.07 & 0.0412 & 0.2804 & 2.78 & 0.4266 & 0.6907 & 0.57 & 0.0665 & 0.3768 & 0.37 \\
\hline & TCDCA & 0.4492 & 0.6109 & 1.47 & 0.0054 & 0.1230 & 2.05 & 0.0067 & 0.0382 & 0.36 & 0.0110 & 0.1873 & 0.4 \\
\hline & GCDCA & 0.1034 & 0.2510 & 1.44 & 0.0072 & 0.1230 & 1.75 & 0.0010 & 0.0197 & 0.43 & 0.0299 & 0.3385 & 0.5 \\
\hline & GCDCS & 0.9960 & 0.9960 & 0.99 & 0.4239 & 0.6766 & 1.08 & 0.0027 & 0.0228 & 0.43 & 0.1727 & 0.5337 & 0.68 \\
\hline \multirow[t]{11}{*}{ Secondary conjugated } & TDCA & 0.0069 & 0.0499 & 2.52 & 0.0546 & 0.3096 & 3.63 & 0.3551 & 0.6907 & 0.37 & 0.0829 & 0.4027 & 0.41 \\
\hline & GDCA & 0.0006 & 0.0196 & 2.81 & 0.0643 & 0.3122 & 2.94 & 0.3384 & 0.6907 & 0.46 & 0.1523 & 0.5337 & 0.55 \\
\hline & TUDCA & 0.7675 & 0.8417 & 0.89 & 0.2373 & 0.5379 & 1.11 & 0.0012 & 0.0197 & 0.41 & 0.0416 & 0.3540 & 0. \\
\hline & GUDCA & 0.6774 & 0.7942 & 1.02 & 0.0878 & 0.3318 & 1.35 & 0.0119 & 0.0578 & 0.38 & 0.2320 & 0.6066 & 0.58 \\
\hline & GDCS & 0.0278 & 0.1182 & 1.63 & 0.2101 & 0.5379 & 2.43 & 0.3898 & 0.6907 & 0.50 & 0.4489 & 0.8475 & 0.51 \\
\hline & TLCA & 0.1744 & 0.3422 & 1.22 & 0.1421 & 0.4391 & 2.00 & 0.1398 & 0.4754 & 0.49 & 0.2738 & 0.6206 & 0.68 \\
\hline & TLCAS & 0.0689 & 0.1952 & 1.39 & 0.2670 & 0.5673 & 2.11 & 0.3551 & 0.6907 & 0.67 & 0.2738 & 0.6206 & 0.4 \\
\hline & GLCA & 0.1909 & 0.3422 & 0.98 & 0.3967 & 0.6766 & 0.99 & 0.4854 & 0.7455 & 0.92 & 0.9062 & 0.9912 & 1.0 \\
\hline & TDCS & 0.7598 & 0.8417 & 1.47 & 0.228 & 0.5379 & 1.41 & 0.0496 & 0.2109 & 0.49 & 0.1623 & 0.5337 & 0.57 \\
\hline & GLCAS & 0.0244 & 0.1182 & 1.37 & 0.5115 & 0.7246 & 1.51 & 0.5269 & 0.7455 & 0.66 & 0.9329 & 0.9912 & 0.7 \\
\hline & GUDCS & 0.4083 & 0.6029 & 0.71 & 0.5748 & 0.7516 & 0.61 & 0.1581 & 0.4886 & 0.52 & 0.7747 & 0.9912 & 1.5 \\
\hline $\begin{array}{l}\text { Bile acid synthesis: } \\
\text { primary/alternative pathway }\end{array}$ & $\mathrm{CA} / \mathrm{CDCA}$ & 0.0058 & 0.0499 & 1.76 & 0.5427 & 0.7381 & 1.26 & 0.4080 & 0.6907 & 1.07 & 0.7747 & 0.9912 & 1.1 \\
\hline \multirow{7}{*}{$\begin{array}{l}\text { Conversion from primary to } s \\
\text { econdary bile acid by the gut } \\
\text { microbiome }\end{array}$} & DCA/CA & 0.6340 & 0.7942 & 1.01 & 0.6761 & 0.7802 & 0.95 & 0.4266 & 0.6907 & 0.74 & 0.8006 & 0.9912 & 0.9 \\
\hline & GDCA/CA & 0.5180 & 0.6773 & 2.16 & 0.5115 & 0.7246 & 4.39 & 0.6613 & 0.7495 & 0.37 & 0.7490 & 0.9912 & 0.3 \\
\hline & TDCA/CA & 0.6628 & 0.7942 & 2.03 & 0.4102 & 0.6766 & 5.28 & 0.6150 & 0.7480 & 0.39 & 0.5333 & 0.9067 & 0.27 \\
\hline & UDCA/CDCA & 0.0838 & 0.2192 & 0.65 & 0.3218 & 0.6436 & 0.77 & 0.3898 & 0.6907 & 0.58 & 0.8796 & 0.9912 & 0.7 \\
\hline & LCA/CDCA & 0.8529 & 0.9062 & 1.13 & 0.6246 & 0.7584 & 1.09 & 0.7827 & 0.8064 & 0.85 & 0.9329 & 0.9912 & 0.7 \\
\hline & GLCA/CDCA & 0.1503 & 0.3408 & 1.00 & 0.7114 & 0.7802 & 1.09 & 0.5701 & 0.7455 & 1.00 & 1.0000 & 1.0000 & 0.9 \\
\hline & TLCA/CDCA & 0.9481 & 0.9768 & 1.28 & 0.6246 & 0.7584 & 2.89 & 0.6380 & 0.7480 & 0.54 & 0.6254 & 0.9912 & 0.52 \\
\hline \multirow{4}{*}{$\begin{array}{l}\text { Glycine or taurine conjugation } \\
\text { of secondary bile acids by } \\
\text { liver enzymes }\end{array}$} & GDCA/DCA & 0.0689 & 0.1952 & 1.20 & 0.1774 & 0.5026 & 2.12 & 0.7828 & 0.8064 & 1.39 & 0.2066 & 0.5853 & 0.54 \\
\hline & TDCA/DCA & 0.2013 & 0.3422 & 1.12 & 0.1179 & 0.4009 & 2.44 & 0.6380 & 0.7480 & 1.49 & 0.1174 & 0.4990 & 0.4 \\
\hline & TUDCA/UDCA & 0.4256 & 0.6029 & 1.49 & 0.7472 & 0.7939 & 2.32 & 0.7090 & 0.7776 & 0.48 & 0.5114 & 0.9067 & 0.50 \\
\hline & GUDCA/UDCA & 0.0674 & 0.1952 & 1.70 & 0.0793 & 0.3318 & 2.50 & 0.5269 & 0.7455 & 0.67 & 0.4094 & 0.8188 & 0.5 \\
\hline
\end{tabular}

To calculate $p$ values the Mann-Whitney $\mathrm{U}$ test was applied. A standard Benjamini-Hochberg method was applied to control the false discovery rate (FDR) for multiple hypothesis testing. $\mathrm{FC}_{1}$ : the ratio of $\mathrm{DN}-\mathrm{PD}$ to $\mathrm{HC}$ in cohort $1 ; \mathrm{FC}_{2}$ : the ratio of DN-PD to $\mathrm{HC}$ in cohort 2; $\mathrm{FC}_{3}$ : the ratio of DO-PD to DN-PD in cohort 2; FC the ratio of CO-PD to DN-PD in cohort 2. CA/CDCA was used to evaluate a possible shift in bile acid biosynthesis form primary to the alternative pathway. DCA/ CA, GDCA/CA, TDCA/CA, UDCA/CDCA, LCA/CDCA, GLCA/CDCA and TLCA/CDCA were calculated to evaluate the enzymatic active of gut microbiome to covert primary bile acids into secondary bile acids. GDCA/DCA, TDCA/DCA, TUDCA/UDCA and GUDCA/UDCA were used to evaluate the enzymatic activity related to taurine and glycine conjugation of secondary bile acids [22]

we applied OPLS-DA to remove the data variation that is independent of categorical variable and decrease the false positive rate. We found that the metabolic profiles of PD, HC and NDC showed a tendency for separation although partially overlapped (Fig. 5a). Six metabolites were identified changed in PD compared to both HC and NDC (Table S8). Besides, we also investigated the impact of antiepileptic medication on the levels of these 


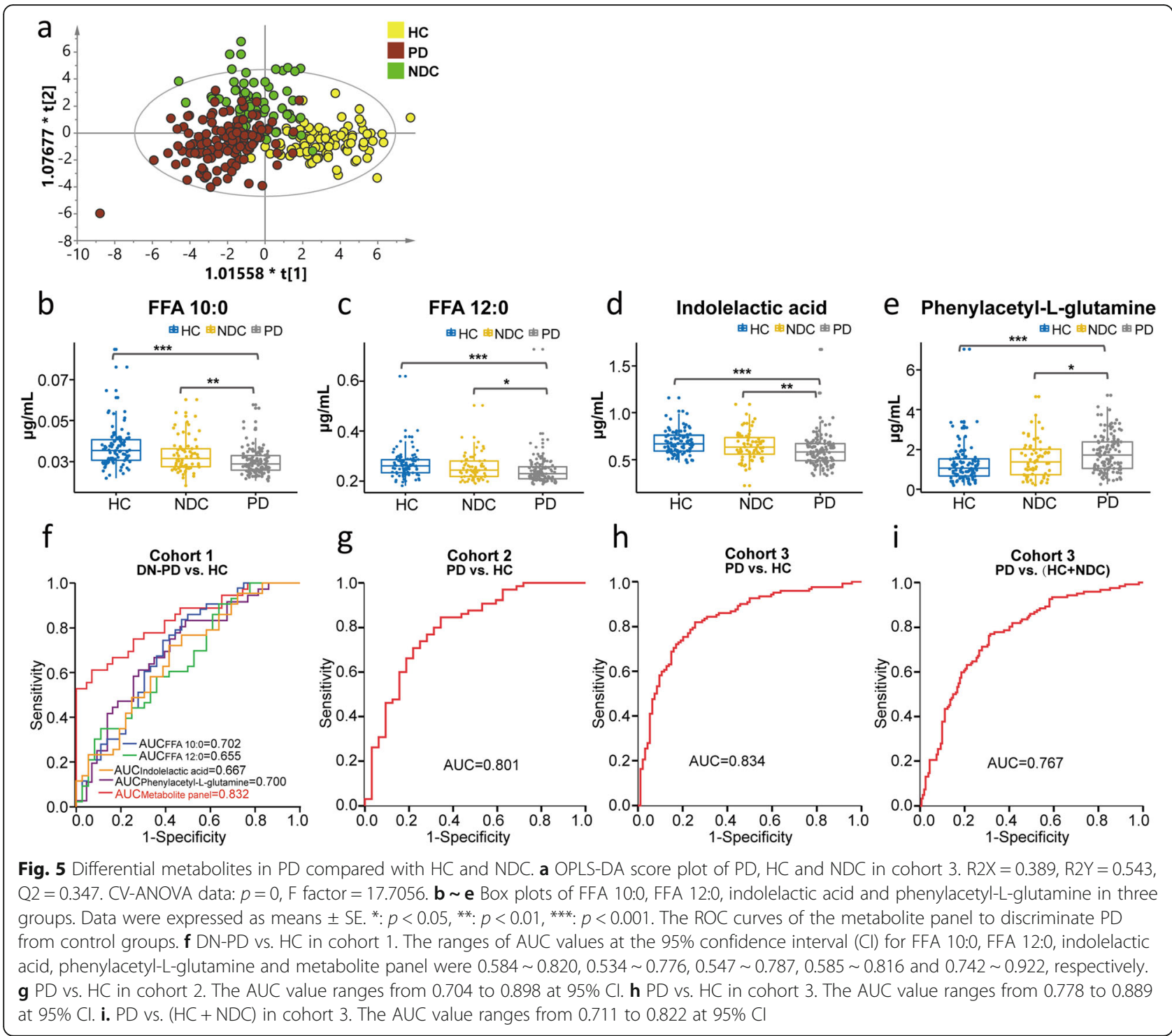

6 metabolites. The results demonstrated that there were no significant differences between treated-epilepsy patients and HC (Table S9). Given that the levels of L-3methoxytyrosine and tyrosine were affected by antiparkinsonian medications, their changes may be induced by antiparkinsonian drugs rather than by PD itself. Thus, they were excluded when developing the discriminant model.

ROC curves of classification models distinguishing PD from $\mathrm{HC}$ using the other 4 metabolites (FFA 10:0, FFA 12: 0 , indolelactic acid and phenylacetyl-glutamine, Fig. 5b-e) were plotted. The AUC values for each metabolite were 0.702, 0.655, 0.667, and 0.700, respectively (Fig. 5f). The discriminant power was improved by combining the 4 metabolites using binary logistic regression analysis with the AUC value reaching to 0.821 . Considering the effects of age on metabolite levels, we also included age as a covariate for the development of diagnostic algorithm. The corresponding ROC curve yielded an AUC value of 0.832 when distinguishing DN-PD from HC (Fig. 5f). To validate the effectiveness of the diagnostic model, we also evaluated its discriminating ability in the other two cohorts. As shown in Fig. 5g-h, the ROC curves produced AUC values of 0.801 and 0.834 when distinguishing $\mathrm{PD}$ from $\mathrm{HC}$ in cohort 2 and cohort 3, respectively. This metabolite panel also showed satisfactory diagnostic performance for distinguishing PD from both $\mathrm{HC}$ and NDC with an AUC value of 0.767 (Fig. 5i). Notably, using the same regression equation developed in cohort 1, the metabolic panel also showed a good discriminability across different cohorts (Figure S5). Detailed parameters of the models are given in Table S10-S13. 


\section{Discussion}

This study presented a comprehensive metabolomic evaluation for PD in 3 independent populations. We investigated the associations of metabolites with gender, age, and stratified PD according to different variables (duration, staging and medication) to better understand the influence of these variables on the biochemical impairment itself. It has been reported that PD patients showed differences between males and females in epidemiological and clinical characteristics, sensitivity to risk factors, and response to treatments [33]. In this study, we found that 24 metabolites including acetylcholine, creatinine, amino acids, PCs and SMs were different between genders in $\mathrm{HC}$ but not in DN-PD, which implied that the reported gender-related differences in PD might be associated with different metabolic reprograming in males and females among PD patients. Besides, we developed a discriminant model consisting of FFA 10:0, FFA 12:0, indolelactic acid and phenylacetylglutamine to assist to diagnose PD. Although previous studies have reported a few discriminant models derived from urine, CSF, and plasma [18, 19, 34-37], the advantage of this study is that the discriminating power of the developed discriminant model was validated by 3 independent cohorts and the same regression equation, which may have great potential for clinical applications in the future. Our study also highlighted that metabolic disturbances in FFA-related metabolism, biosynthesis of BA and steroid hormone, and amino acid metabolism might be involved in PD pathogenesis.

Until recently, alteration of FFA metabolism has been increasingly recognized in PD [5, 38-40]. We found that metabolism of unsaturated FFAs, especially linoleic acid, linolenic acid, and arachidonic acid metabolism, were remarkably perturbed in PD (Fig. 6a). Generally, FFAs are considered to be required for membrane formation, signaling molecule generation and energy supply through beta-oxidation [41]. Recent reports suggested that FFAs, particularly long-chain polyunsaturated FFAs (PUFAs), can bind to monomeric $\alpha$-synuclein and accelerate the formation of $\alpha$-synuclein assemblies [42, 43]. It has been reported that fatty acid-binding protein 3 (FABP3) was elevated in the cerebrospinal fluid (CSF) and serum of PD patients and highly expressed in the dopaminergic neurons $[44,45]$, and was able to promote $\alpha$-synuclein oligomerization in cultured dopaminergic neurons [42]. Considering this, targeting FABP3 may represent an attractive therapeutic strategy for PD. Prevous study has demonstrated that the developed FABP3 ligands can inhibit arachidonic acid-induced $\alpha$-synuclein oligomerization in neuro-2A cells [46]. Epoxy fatty acids (EpFAs), the oxidized metabolites of PUFAs, has been found to have potent anti-inflammatory properties [47]. EpFAs can be further metabolized into corresponding diols by soluble epoxide hydrolase $(\mathrm{sEH})$ [48]. It has been reported that overexpression of $\mathrm{sEH}$ in the striatum significantly enhanced MPTPinduced neurotoxicity [49]. Diminished PUFAs in PD may be due to the enhancement of their downstream metabolic flux. Therefore, targeting EpFA metabolism may provide novel insight into PD etiology.

To date, a few studies have implicated BAs in PD and mainly focused on the neuroprotection of UDCA and its derivatives $[50,51]$. It has been reported that UDCA or TUDCA treatment could improve motor performance, ameliorate mitochondrial dysfunction and neuroinflammation and prevent the decline of striatal dopamine content in various PD models [5052]. However, knowledge of the potential efficacy of other BAs in PD are still limited. Our study presented a comprehensive analysis of BA profiles in PD patients and identified a range of conjugated and unconjugated BAs, which were significantly disturbed in PD (Fig. 6b). A shift in initial cholesterol metabolism from alternative pathway to primary pathway suggested that altered enzymatic activities lead to excess production of BAs, many of which might be cytotoxic [53]. A previous study has reported reductions of neuroprotective BAs in a prodromal PD mouse model [32]. Together with our findings, it seems that disturbances in BA metabolism might play important roles in the development of PD, even in prodromal stages. Alterations of secondary BAs and BA ratios indicated possible alterations in enzymatic activities of gut microbiota. Notably, the elevations of BAs and BA ratios tend to be alleviated after L-dopa treatment. Increased Firmicutes has been found in PD patients [54]. It has been reported that several species of Firmicutes in gut can dehydroxylate CA to form DCA, which might be toxic to cells [55]. Determining the specific role and its precise molecular mechanism of each BA in PD might provide novel cues for future therapeutic strategies.

Recently, enteric dysbacteriosis has been recognized as a consistent feature of PD [56]. Abnormalities in the composition and distribution of intestinal bacteria have been suggested in PD patients [57]. It has been postulated that $\alpha$-synuclein pathology may spread from gut to the brain and contribute to PD etiology, however, the exact mechanism remains unclear [13]. The microbiota-derived metabolites provide a functional readout of the microbiome and can indicate the metabolic interplay among the host, diet, and intestinal bacteria [58]. Apart from BAs, we found a list of microbiota-derived metabolites including proteolytic metabolism products and tryptophan catabolites, which showed significant alterations in PD (Fig. 6c-d). Elevated $p$-cresol sulfate has been detected in the CSF 


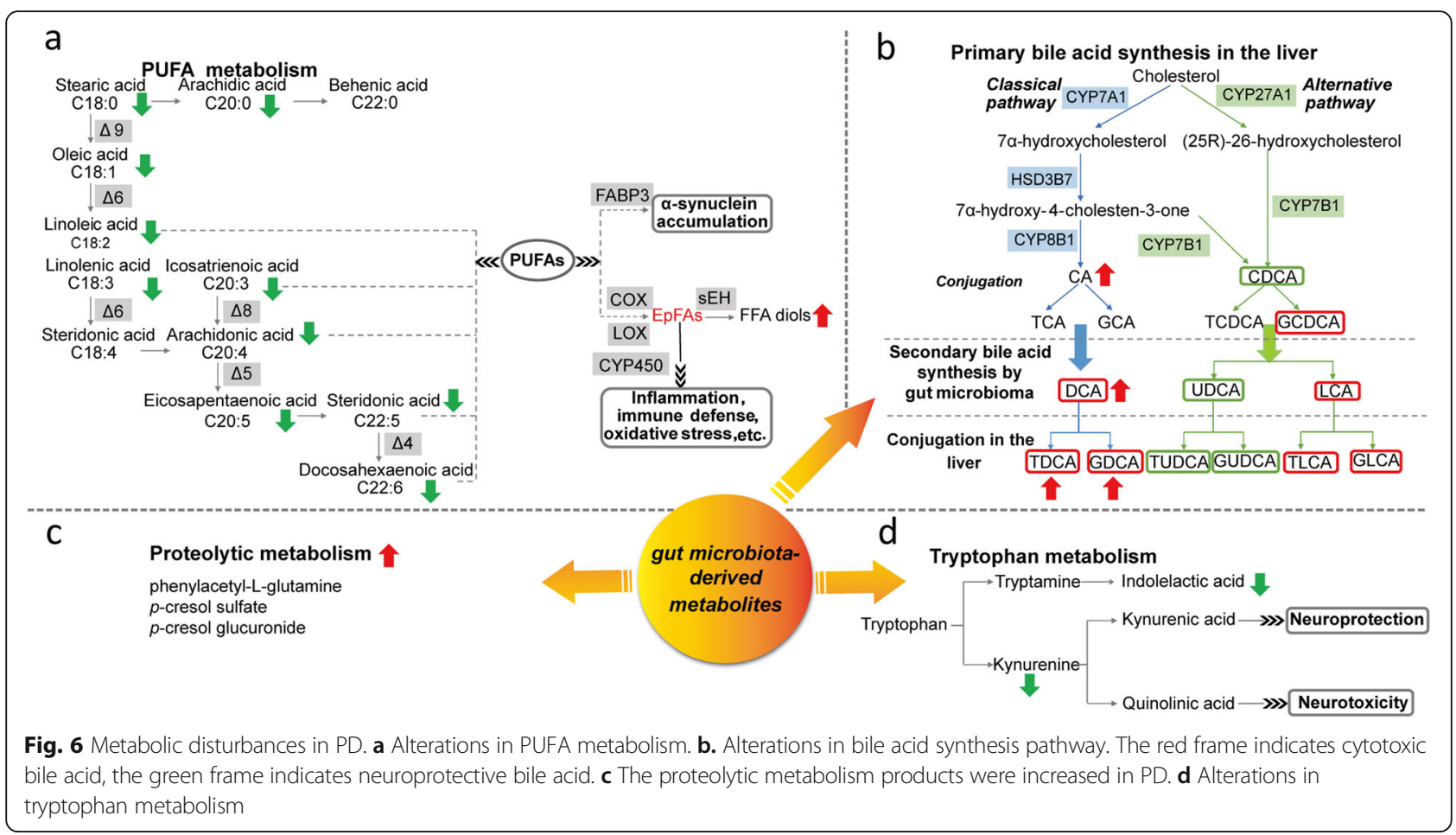

of PD [39, 59]. Recently, Mihai and colleagues demonstrated that $p$-cresol sulfate and phenylacetyl-Lglutamine were increased in the serum of PD and the elevation of these deleterious metabolites was positively correlated with firmer stool and constipation severity among patients [56]. Our study further confirms the increased production of $p$-cresol sulfate and phenylacetyl-L-glutamine in the plasma of PD. Moreover, we identified a novel $p$-cresol metabolite, $p$-cresol glucuronide, a byproduct of protein degradation by gut bacteria, showing significantly increased level in PD. It was revealed that $p$-cresol could inhibit the oxidative respiration and proliferation of colonoscopy cells [56, 60]. Using radio-opaque markers, a study investigated the relationship between colonic transit time and human colonic metabolism and concluded that delayed transit time is accompanied by a shift in colonic metabolism from carbohydrate fermentation to protein catabolism [61]. Therefore, this metabolic alteration may be mechanistically relevant to gastrointestinal disorders in PD patients $[62,63]$. A few studies have indicated that the imbalance of kynurenine (Kyn) metabolism, the main catabolic rote of tryptophan, played important roles in PD pathogenesis $[19,64]$. Lower levels of KA and KA/Kyn ratio and higher levels of $\mathrm{QA}$ and QA/KA ratio were reported in the plasma of PD, indicating a biased Kyn pathway toward producing oxidative stress and excitotoxicity [19]. Apart from Kyn metabolites, we also observed a significantly decreased level of indolelactic acid, another tryptophan catabolite, in PD. Indolelactic acid can be produced by several Clostridium species such as Clostridium sporogenes and Clostridium saccharolyticum [65, 66]. Decreased Clostridium saccharolyticum has been reported in fecal samples of PD patients [54]. Besides, alterations of several other Clostridium species and Bacteroides species that involved in tryptophan catabolism were also observed in PD [54, 67]. A recent study demonstrated that gut microbiota-derived tryptophan catabolites could modulate inflammatory response by attenuating the release of pro-inflammatory cytokines and the cytokine-mediated upregulation of lipogenesis in macrophages and hepatocytes [68]. Previous report also showed anti-neuroinflammatory activities of indole alkaloids in lipopolysaccharide-stimulated BV2 microglial cells [69]. The decreased level of indolelactic acid may contribute to promoting the inflammation in PD.

Both epidemiological and clinical studies have consistently reported that caffeine consumption could reduce the risk of PD [16, 70]. We found significant reduction of its downstream metabolite (trigonelline) in the plasma of PD despite an equivalent caffeine intake to controls. Although there is no statistical significance, we also observed a decreased level of caffeine in PD. Previous studies also reported reduced levels of caffeine and its metabolites in PD and indicated that the reductions have 
no associations with daily caffeine consumption [3, 71]. A recent work reported that the decrease of these metabolites was also observed in patients with glial cytoplasmic inclusions and neuronal tau accumulation [72]. A common mechanism such as malabsorption from small intestine or abnormal clearance of caffeine may underlie these parkinsonian disorders [72].

Additionally, we documented increased production of cortisol and corticosterone and decreased aldosterone in PD. In the literature, it has been revealed an elevation of cortisol in the plasma and saliva of PD patients [73, 74]. High levels of cortisol can damage substantia nigra striatum system and temporarily aggravate the motor and neuropsychiatric symptoms of PD patients $[75,76]$. Corticosterone, the other important glucocorticoid, has been found to impair learning and memory function and cause calciuminduced neurotoxicity in several PD models [77-79]. A recent study indicated that genes related to aldosterone synthesis and secretion were altered in PD [80]. The renin-angiotensin-aldosterone system (RAS) is crucial in the development of hypertension and organ damage, and the activation of brain RAS has been revealed to aggravate the cognitive decline and dopaminergic neuron loss by promoting oxidative stress and inflammation processes [81, 82].

This study provides a comprehensive analysis of metabolic reprogramming in PD. However, it has several limitations. Firstly, PD was diagnosed based on clinical criteria without laboratory confirmation. Further studies to link peripheral metabolic changes to pathophysiology markers, genetic findings and neuroimaging profiles are recommended. Secondly, we only investigated the effects of several commonly used antiparkinsonian treatments, the impacts of other medications cannot be clarified. There are quite few factors such as genetic background, disease history, lifestyle, and diet, etc. which might influence the profiles of the metabolites in PD and controls. To address this issue, future study is necessary to calibrate the levels of metabolites with these factors in a larger cohort investigation.

\section{Conclusions}

In summary, we highlighted that metabolic disturbances in PUFA metabolism, BA and steroid hormone biosynthesis, caffeine metabolism and amino acid metabolism are crucial metabolic events underlying PD. The accumulated microbiota-derived deleterious metabolites including $p$-cresol sulfate, $p$-cresol glucuronide and phenylacetyl-L-glutamine implied an important role of intestinal homeostasis in downstream neurodegenerative processes. These evaluations could improve our understanding of PD etiopathogenesis and facilitate target screening for therapeutic intervention.

\section{Supplementary Information}

The online version contains supplementary material available at https://doi. org/10.1186/s13024-021-00425-8.

Additional file 1: Table S1. Concentrations of the stable isotope labeled internal standards in methanol. Table S2. Statistical results of FFAs in blank and analytical samples. Table S3. Statistical results of differential metabolites between male and female in HC group. Table S4. Differential metabolites accountable for the discrimination between drug-naïve PD patients and controls. Table S5. Associations between the differential metabolites and disease severity. Table S6. Associations between the differential metabolites and duration time. Table S7. Associations between the differential metabolites and age. Table S8. Statistical results of differential metabolites in PD compared with both $\mathrm{HC}$ and NDC groups in cohort 3. Table S9. Statistical results of the six selected differential metabolites in treated-epilepsy patients and HC. Table S10. Parameters of the binary logistic regression model in cohort 1. Table S11. Parameters of the binary logistic regression model in cohort 2. Table S12. Parameters of the binary logistic regression model in cohort 3 (PD vs. $\mathrm{HC}+\mathrm{NDC}$ ). Table S13. Parameters of the binary logistic regression model in cohort 3 (PD vs. HC). Figure S1. Robust assessment of the analytical method across three independent cohorts. Figure S2. PCA analysis of the metabolic profiles in male and female of drug-naïve PD and HC. Figure S3. Permutation test (999 times) of the PLS-DA models. Figure S4. Pathway analysis of the differential metabolites in drug-naïve PD compared with HC. Figure S5. The ROC curves of the metabolite panel to discriminate PD from control groups across different cohorts based on the regression equation developed in cohort 1.

\section{Abbreviations}

QA: Quinolinic acid; KA: Kynurenic acid; BA: Bile acid; HC: Healthy control; NDC: Neurological disease control; IS: Internal standard; QC: Quality control; RSD: Relative standard deviation; PCA: Principal component analysis; PLSDA: Partial least square discriminant analysis; OPLS-DA: Orthogonal PLS-DA; FDR: False discovery rate; ROC: Receiver operating characteristic; AUC: The area under the curve; DN-PD: Drug-naive PD; PC: Phosphatidylcholine; SM: Sphingomyelin; FFA: Fatty acid; FFAD: FFA amide; DO-PD: L-dopa-treated PD; PR-PD: Pramipexole-treated PD; CO-PD: The combination of L-dopa and pramipexole-treated PD; LPC: Lysophosphatidylcholine; PUFA: Polyunsaturated FFA; FABP3: Fatty acid-binding protein 3; CSF: Cerebrospinal fluid; EpFAs: Epoxy fatty acids; sEH: soluble epoxide hydrolase; RAS: Renin-angiotensin-aldosterone system; Kyn: Kynurenine; LOX: Lipoxygenase; COX: Cyclooxygenases; CA: Cholic acid;

CDCA: Chenodeoxycholic acid; DCA: Deoxycholic acid;

UDCA: Ursodeoxycholic acid; LCA: Lithocholic acid; GCA: Glycocholic acid; TCA: Taurocholic acid; TCAS: Taurocholic acid 3-sulfate;

TCDCA: Taurochenodeoxycholic acid; GCDCA: Glycochenodeoxycholic acid; GCDCS: Glycochenodeoxycholic acid 3-sulfate; TDCA: Taurodeoxycholic acid; GDCA: Glycodeoxycholic acid; TUDCA: Tauroursodeoxycholic acid;

GUDCA: Glycoursodeoxycholic acid; GDCS: Glycodeoxycholic acid 3-sulfate; TLCA: Taurolithocholic acid; TLCAS: Taurolithocholic acid 3-sulfate;

GLCA: Glycolithocholic acid; TDCS: Taurodeoxycholic acid 3-sulfate; GLCA

S: Glycolithocholic acid 3-sulfate; GUDCS: Glycoursodeoxycholic acid 3-sulfate

\section{Acknowledgements}

We thank all the participants and their families for their commitments and statements to help advance research into clinical diagnosis of PD. We would like to thank Professor Jau-Shyong Hong, from Neurobiology Laboratory National Institute of Environmental Health Sciences, National Institutes of Health to give us a critical review and advices for this manuscript.

\section{Authors' contributions}

WDL and GWX designed the research; YPS, ZYL performed the study; YPS, ZYL and XLW analyzed the data; YPS wrote the manuscript; TBL and XJX collected the clinical samples; SL, GWX and WDL revised the manuscript. All authors read and approved the final manuscript.

\section{Funding}

This work was supported by National Natural Science Foundation of China (NSFC 81771521, 21876169 and 22004013), the National Key Research and 
Development Program of China (2016YFC1306600) and Key Realm R\&D Program of Guangdong Province (2018B030337001).

\section{Availability of data and materials}

The datasets used and/or analyzed during the current study are available from the corresponding author on reasonable request.

\section{Ethics approval and consent to participate}

This study was approved by Ethics Committee of the First Affiliated Hospital of Dalian Medical University and all subjects or their legally authorized caregivers signed informed consents.

\section{Consent for publication}

Not applicable.

\section{Competing interests}

The author declares no competing interests.

\section{Author details}

'Center for Clinical Research on Neurological Diseases, The First Affiliated Hospital, Dalian Medical University, 193 Lianhe Road, Dalian, China. 'Liaoning Provincial Key Laboratory for Research on the Pathogenic Mechanisms of Neurological Diseases, The First Affiliated Hospital, Dalian Medical University, 193 Lianhe Road, Dalian, China. ${ }^{3}$ CAS Key Laboratory of Separation Science for Analytical Chemistry, Dalian Institute of Chemical Physics, Chinese Academy of Sciences, 457 Zhongshan Road, Dalian 116023, China. ${ }^{4}$ Institute of Neurology, Sichuan Academy of Medical Science-Sichuan Provincial Hospital, Medical School of UESTC, Sichuan, China.

\section{Received: 14 October 2020 Accepted: 11 January 2021}

\section{Published online: 23 January 202}

\section{References}

1. de Lau LM, Breteler MM. Epidemiology of Parkinson's disease. Lancet Neurol. 2006;5:525-35.

2. Tysnes OB, Storstein A. Epidemiology of Parkinson's disease. J Neural Transm (Vienna). 2017:124:901-5.

3. Hatano T, Saiki S, Okuzumi A, Mohney RP, Hattori N. Identification of novel biomarkers for Parkinson's disease by metabolomic technologies. J Neurol Neurosurg Psychiatry. 2016;87:295-301.

4. Cressatti M, Juwara L, Galindez JM, Velly AM, Nkurunziza ES, Marier S, Canie O, Gornistky M, Schipper HM. Salivary microR-153 and microR-223 levels as potential diagnostic biomarkers of idiopathic Parkinson's disease. Mov Disord. 2020;35:468-77.

5. Burte F, Houghton D, Lowes H, Pyle A, Nesbitt S, Yarnall A, Yu-Wai-Man P, Burn DJ, Santibanez-Koref M, Hudson G. Metabolic profiling of Parkinson's disease and mild cognitive impairment. Mov Disord. 2017;32:927-32.

6. Li T, Yang Z, Li S, Cheng C, Shen B, Le W. Alterations of NURR1 and cytokines in the peripheral blood mononuclear cells: combined biomarkers for Parkinson's disease. Front Aging Neurosci. 2018;10:392.

7. Le W, Pan T, Huang M, Xu P, Xie W, Zhu W, Zhang X, Deng H, Jankovic J. Decreased NURR1 gene expression in patients with Parkinson's disease. J Neurol Sci. 2008:273:29-33.

8. Yang Z, Li T, Cui Y, Li S, Cheng C, Shen B, Le W. Elevated plasma microRNA105-5p level in patients with idiopathic Parkinson's disease: a potential disease biomarker. Front Neurosci. 2019;13:218

9. Powers R, Lei S, Anandhan A, Marshall DD, Worley B, Cerny RL, Dodds ED, Huang Y, Panayiotidis MI, Pappa A, Franco R. Metabolic investigations of the molecular mechanisms associated with Parkinson's disease. Metabolites. 2017;7:22.

10. Shao $Y$, Le $W$. Recent advances and perspectives of metabolomics-based investigations in Parkinson's disease. Mol Neurodegener. 2019;14:3.

11. Picca A, Calvani R, Landi G, Marini F, Biancolillo A, Gervasoni J, Persichilli S, Primiano A, Urbani A, Bossola M, et al. Circulating amino acid signature in older people with Parkinson's disease: a metabolic complement to the EXosomes in PArkiNson disease (EXPAND) study. Exp Gerontol. 2019;128: 110766

12. Nho K, Kueider-Paisley A, MahmoudianDehkordi S, Arnold M, Risacher SL, Louie G, Blach C, Baillie R, Han X, Kastenmuller G, et al. Altered bile acid profile in mild cognitive impairment and Alzheimer's disease: relationship to neuroimaging and CSF biomarkers. Alzheimers Dement. 2019;15:232-44.
13. van Kessel SP, El Aidy S. Bacterial metabolites Mirror altered gut microbiota composition in patients with Parkinson's disease. J Parkinsons Dis. 2019;9: S359-S70.

14. Jawaid A, Khan R, Polymenidou M, Schulz PE. Disease-modifying effects of metabolic perturbations in ALS/FTLD. Mol Neurodegener. 2018;13:63.

15. Ren S, Shao Y, Zhao X, Hong CS, Wang F, Lu X, Li J, Ye G, Yan M, Zhuang Z, et al. Integration of metabolomics and Transcriptomics reveals major metabolic pathways and potential biomarker involved in prostate cancer. Mol Cell Proteomics. 2016;15:154-63.

16. Han W, Sapkota S, Camicioli R, Dixon RA, Li L. Profiling novel metabolic biomarkers for Parkinson's disease using in-depth metabolomic analysis. Mov Disord. 2017:32:1720-8.

17. Masato A, Plotegher N, Boassa D, Bubacco L. Impaired dopamine metabolism in Parkinson's disease pathogenesis. Mol Neurodegener. 2019;14:35.

18. Saiki S, Hatano T, Fujimaki M, Ishikawa Kl, Mori A, Oji Y, Okuzumi A, Fukuhara T, Koinuma T, Imamichi Y, et al. Decreased long-chain acylcarnitines from insufficient beta-oxidation as potential early diagnostic markers for Parkinson's disease. Sci Rep. 2017;7:7328.

19. Chang KH, Cheng ML, Tang HY, Huang CY, Wu YR, Chen CM. Alternations of metabolic profile and Kynurenine metabolism in the plasma of Parkinson's disease. Mol Neurobiol. 2018;55:6319-28.

20. Schulte EC, Altmaier E, Berger HS, Do KT, Kastenmuller G, Wahl S, Adamski J, Peters A, Krumsiek J, Suhre K, et al. Alterations in lipid and inositol metabolisms in two dopaminergic disorders. PLoS One. 2016;11:e0147129.

21. Li T, Chiang JY. Bile acid signaling in metabolic disease and drug therapy. Pharmacol Rev. 2014;66:948-83.

22. MahmoudianDehkordi S, Arnold M, Nho K, Ahmad S, Jia W, Xie G, Louie G, Kueider-Paisley A, Moseley MA, Thompson JW, et al. Altered bile acid profile associates with cognitive impairment in Alzheimer's disease-an emerging role for gut microbiome. Alzheimers Dement. 2019;15:76-92.

23. Kiriyama $Y$, Nochi $H$. The biosynthesis, signaling, and neurological functions of bile acids. Biomolecules. 2019;9:232.

24. Postuma RB, Berg D, Stern M, Poewe W, Olanow CW, Oertel W, Obeso J, Marek K, Litvan I, Lang AE, et al. MDS clinical diagnostic criteria for Parkinson's disease. Mov Disord. 2015;30:1591-601.

25. Huang $Y$, Chen G, Liu X, Shao Y, Gao P, Xin C, Cui Z, Zhao X, Xu G. Serum metabolomics study and eicosanoid analysis of childhood atopic dermatitis based on liquid chromatography-mass spectrometry. J Proteome Res. 2014; 13:5715-23.

26. Smilde AK, van der Werf MJ, Bijlsma S, van der Werff-van der Vat BJC, Jellema RH. Fusion of mass spectrometry-based metabolomics data. Anal Chem. 2005;77:6729-36

27. Zhao X, Zeng Z, Chen A, Lu X, Zhao C, Hu C, Zhou L, Liu X, Wang X, Hou X, et al. Comprehensive strategy to construct in-house database for accurate and batch identification of Small molecular metabolites. Anal Chem. 2018; 90:7635-43

28. Benjamini $Y$, Hochberg $Y$. Controlling the false discovery rate : a practical and powerful approach to multiple testing. J R Stat Soc. 1995;57:289-300.

29. Schisterman EF, Perkins NJ, Liu A, Bondell H. Optimal cut-point and its corresponding Youden index to discriminate individuals using pooled blood samples. Epidemiology. 2005;16:73-81.

30. Yao CH, Liu GY, Yang K, Gross RW, Patti GJ. Inaccurate quantitation of palmitate in metabolomics and isotope tracer studies due to plastics. Metabolomics. 2016;12:143.

31. Goetz CG, Poewe W, Rascol O, Sampaio C, Stebbins GT, Counsell C, Giladi N, Holloway RG, Moore CG, Wenning GK, et al. Movement Disorder Society task force report on the Hoehn and Yahr staging scale: status and recommendations the Movement Disorder Society task force on rating scales for Parkinson's disease. Mov Disord. 2004;19:1020-8.

32. Graham SF, Rey NL, Ugur Z, Yilmaz A, Sherman E, Maddens M, BahadoSingh RO, Becker K, Schulz E, Meyerdirk LK, et al. Metabolomic profiling of bile acids in an experimental model of prodromal Parkinson's disease. Metabolites. 2018;8:71.

33. Miller IN, Cronin-Golomb A. Gender differences in Parkinson's disease: clinical characteristics and cognition. Mov Disord. 2010;25:2695-703.

34. Luan H, Liu LF, Tang Z, Mok VC, Li M, Cai Z. Elevated excretion of biopyrrin as a new marker for idiopathic Parkinson's disease. Parkinsonism Relat Disord. 2015:21:1371-2.

35. Luan H, Liu LF, Meng N, Tang Z, Chua KK, Chen LL, Song JX, Mok VC, Xie LX, Li M, Cai Z. LC-MS-based urinary metabolite signatures in idiopathic Parkinson's disease. J Proteome Res. 2015;14:467-78. 
36. Luan H, Liu L-F, Tang Z, Zhang M, Chua K-K, Song J-X, Mok VCT, Li M, Cai Z. Comprehensive urinary metabolomic profiling and identification of potential noninvasive marker for idiopathic Parkinson's disease. Sci Rep. 2015:5:13888.

37. Andersen AD, Blaabjerg M, Binzer M, Kamal A, Thagesen H, Kjaer TW, Stenager E, Gramsbergen JBP. Cerebrospinal fluid levels of catecholamines and its metabolites in Parkinson's disease: effect of IDOPA treatment and changes in levodopa-induced dyskinesia. J Neurochem. 2017;141:614-25.

38. Ueno SI, Saiki S, Fujimaki M, Takeshige-Amano H, Hatano T, Oyama G, Ishikawa Kl, Yamaguchi A, Nojiri S, Akamatsu W, Hattori N. Zonisamide administration improves fatty acid beta-oxidation in Parkinson's disease. Cells. 2018;8:14

39. Lucio M, Willkommen D, Schroeter M, Sigaroudi A, Schmitt-Kopplin P, Michalke B. Integrative Metabolomic and Metallomic analysis in a casecontrol cohort with Parkinson's disease. Front Aging Neurosci. 2019;11:331.

40. Shah A, Han P, Wong MY, Chang RC, Legido-Quigley C. Palmitate and stearate are increased in the plasma in a 6-OHDA model of Parkinson's disease. Metabolites. 2019;9:31.

41. Currie E, Schulze A, Zechner R, Walther TC, Farese RV Jr. Cellular fatty acid metabolism and cancer. Cell Metab. 2013;18:153-61.

42. Kawahata I, Bousset L, Melki R, Fukunaga K. Fatty acid-binding protein 3 is critical for alpha-Synuclein uptake and MPP(+)-induced mitochondrial dysfunction in cultured dopaminergic neurons. Int J Mol Sci. 2019;20:5358.

43. Sharon R, Bar-Joseph I, Frosch MP, Walsh DM, Hamilton JA, Selkoe DJ. The Formation of Highly Soluble Oligomers of \&\#x3b1;-Synuclein Is Regulated by Fatty Acids and Enhanced in Parkinson's Disease. Neuron. 2003:37:583-95.

44. Mollenhauer B, Steinacker P, Bahn E, Bibl M, Brechlin P, Schlossmacher MG, Locascio JJ, Wiltfang J, Kretzschmar HA, Poser S, et al. Serum heart-type fatty acid-binding protein and cerebrospinal fluid tau: marker candidates for dementia with Lewy bodies. Neurodegener Dis. 2007;4:366-75.

45. Shioda N, Yamamoto Y, Watanabe M, Binas B, Owada Y, Fukunaga K. Hearttype fatty acid binding protein regulates dopamine D2 receptor function in mouse brain. J Neurosci. 2010; 30:3146-55.

46. Cheng A, Shinoda Y, Yamamoto T, Miyachi H, Fukunaga K. Development of FABP3 ligands that inhibit arachidonic acid-induced a-synuclein oligomerization. Brain Res. 2019;1707:190-7.

47. Wasternack C, Feussner I. The Oxylipin pathways: biochemistry and function. Annu Rev Plant Biol. 2018;69:363-86.

48. Atone J, Wagner K, Hashimoto K, Hammock BD. Prostaglandins and other lipid mediators cytochrome P450 derived epoxidized fatty acids as a therapeutic tool against neuroinflammatory diseases. Prostaglandins Other Lipid Mediat. 2019;147:106385.

49. Ren Q, Ma M, Yang J, Nonaka R, Yamaguchi A, Ishikawa K-I, Kobayashi K, Murayama S, Hwang SH, Saiki S, et al. Soluble epoxide hydrolase plays a key role in the pathogenesis of Parkinson's disease. Proceed Nat Acad Sci. 2018; 115:E5815-E23.

50. Rosa Al, Duarte-Silva S, Silva-Fernandes A, Nunes MJ, Carvalho AN, Rodrigues E, Gama MJ, Rodrigues CMP, Maciel P, Castro-Caldas M. Tauroursodeoxycholic acid improves motor symptoms in a mouse model of Parkinson's disease. Mol Neurobiol. 2018;55:9139-55.

51. Abdelkader NF, Safar MM, Salem HA. Ursodeoxycholic acid ameliorates apoptotic Cascade in the rotenone model of Parkinson's disease: modulation of mitochondrial perturbations. Mol Neurobiol. 2016;53:810-7.

52. Mortiboys H, Furmston R, Bronstad G, Aasly J, Elliott C, Bandmann O. UDCA exerts beneficial effect on mitochondrial dysfunction in LRRK2(G2019S) carriers and in vivo. Neurology. 2015;85:846-52.

53. Barrasa JI, Olmo N, Pérez-Ramos P, Santiago-Gómez A, Lecona E, Turnay J, Antonia LM. Deoxycholic and chenodeoxycholic bile acids induce apoptosis via oxidative stress in human colon adenocarcinoma cells. Apoptosis. 2011; 16:1054.

54. Bedarf JR, Hildebrand F, Coelho LP, Sunagawa S, Bahram M, Goeser F, Bork $P$, Wullner U. Functional implications of microbial and viral gut metagenome changes in early stage L-DOPA-naive Parkinson's disease patients. Genome Med. 2017;9:39.

55. Donia MS, Fischbach MA. HUMAN MICROBIOTA. Small molecules from the human microbiota. Science. 2015;349:1254766.

56. Cirstea MS, Yu AC, Golz E, Sundvick K, Kliger D, Radisavljevic N, Foulger LH, Mackenzie M, Huan T, Finlay BB, Appel-Cresswell S. Microbiota Composition and Metabolism Are Associated With Gut Function in Parkinson's Disease. Movement Disorders; 2020.
57. Lin A, Zheng W, He Y, Tang W, Wei X, He R, Huang W, Su Y, Huang Y, Zhou $\mathrm{H}$, Xie H. Gut microbiota in patients with Parkinson's disease in southern China. Parkinsonism Relat Disord. 2018;53:82-8.

58. Zierer J, Jackson MA, Kastenmuller G, Mangino M, Long T, Telenti A, Mohney RP, Small KS, Bell JT, Steves CJ, et al. The fecal metabolome as a functional readout of the gut microbiome. Nat Genet. 2018;50:790-5.

59. Sankowski B, Księżarczyk K, Raćkowska E, Szlufik S, Koziorowski D, Giebultowicz J. Higher cerebrospinal fluid to plasma ratio of p-cresol sulfate and indoxyl sulfate in patients with Parkinson's disease. Clin Chim Acta. 2020;501:165-73.

60. Diether N, Willing B. Microbial fermentation of dietary protein: an important factor in diet-microbe-host interaction. Microorganisms. 2019;7:19.

61. Roager HM, Hansen LB, Bahl MI, Frandsen HL, Carvalho V, Gøbel RJ, Dalgaard MD, Plichta DR, Sparholt MH, Vestergaard H, et al. Colonic transit time is related to bacterial metabolism and mucosal turnover in the gut. Nat Microbiol. 2016;1:16093.

62. Sun MF, Shen YQ. Dysbiosis of gut microbiota and microbial metabolites in Parkinson's disease. Ageing Res Rev. 2018;45:53-61.

63. Scheperjans F, Aho V, Pereira PA, Koskinen K, Paulin L, Pekkonen E, Haapaniemi E, Kaakkola S, Eerola-Rautio J, Pohja M, et al. Gut microbiota are related to Parkinson's disease and clinical phenotype. Mov Disord. 2015;30: 350-8.

64. Havelund JF, Andersen AD, Binzer M, Blaabjerg M, Heegaard NHH, Stenager E, Faergeman NJ, Gramsbergen JB. Changes in kynurenine pathway metabolism in Parkinson patients with L-DOPA-induced dyskinesia. J Neurochem. 2017;142:756-66.

65. Dodd D, Spitzer MH, Van Treuren W, Merrill BD, Hryckowian AJ, Higginbottom SK, Le A, Cowan TM, Nolan GP, Fischbach MA, Sonnenburg JL. A gut bacterial pathway metabolizes aromatic amino acids into nine circulating metabolites. Nature. 2017;551:648-52

66. Roager HM, Licht TR. Microbial tryptophan catabolites in health and disease. Nat Commun. 2018;9:3294

67. Hasegawa S, Goto S, Tsuji H, Okuno T, Asahara T, Nomoto K, Shibata A, Fujisawa Y, Minato T, Okamoto A, et al. Intestinal Dysbiosis and lowered serum lipopolysaccharide-binding protein in Parkinson's disease. PLoS One. 2015;10:e0142164.

68. Krishnan S, Ding Y, Saedi N, Choi M, Sridharan GV, Sherr DH, Yarmush ML, Alaniz RC, Jayaraman A, Lee K. Gut microbiota-derived tryptophan metabolites modulate inflammatory response in hepatocytes and macrophages. Cell Rep. 2018;23:1099-111.

69. Kim DC, Quang TH, Yoon C-S, Ngan NTT, Lim S-I, Lee S-Y, Kim Y-C, Oh H. Anti-neuroinflammatory activities of indole alkaloids from kanjang (Korean fermented soy source) in lipopolysaccharide-induced BV2 microglial cells. Food Chem. 2016; 213:69-75.

70. Ascherio A, Schwarzschild MA. The epidemiology of Parkinson's disease: risk factors and prevention. Lancet Neurol. 2016;15:1257-72.

71. Fujimaki M, Saiki S, Li Y, Kaga N, Taka H, Hatano T, Ishikawa K-I, Oji Y, Mori A, Okuzumi A, et al. Serum caffeine and metabolites are reliable biomarkers of early Parkinson disease. Neurology. 2018;90:e404-e11.

72. Takeshige-Amano H, Saiki S, Fujimaki M, Ueno SI, Li Y, Hatano T, Ishikawa Kl, Oji Y, Mori A, Okuzumi A, et al. Shared metabolic profile of caffeine in Parkinsonian disorders. Mov Disord. 2020;35:1438-47.

73. Costa CM, Oliveira GL, Fonseca ACS, Lana RC, Polese JC, Pernambuco AP. Levels of cortisol and neurotrophic factor brain-derived in Parkinson's disease. Neurosci Lett. 2019;708:134359.

74. Soares NM, Pereira GM, Altmann V, de Almeida RMM, Rieder CRM. Cortisol levels, motor, cognitive and behavioral symptoms in Parkinson's disease: a systematic review. J Neural Transm. 2018;126:219-32.

75. Kim ST, Ji HC, Jin WC, Kim SW, Hwang O. Immobilization stress causes increases in tetrahydrobiopterin, dopamine, and neuromelanin and oxidative damage in the nigrostriatal system. J Neurochem. 2005;95:89-98.

76. Ruzicka F, Jech R, Novakova L, Urgosik D, Bezdicek O, Vymazal J, Ruzicka E. Chronic stress-like syndrome as a consequence of medial site subthalamic stimulation in Parkinson's disease. Psychoneuroendocrinology. 2015;52:302-10.

77. Alam M, Schmidt W. Mitochondrial complex I inhibition depletes plasma testosterone in the rotenone model of Parkinson's disease. Physiol Behav. 2004:83:395-400.

78. Mesches MH, Fleshner M, Heman KL, Rose GM, Diamond DM. Exposing rats to a predator blocks primed burst potentiation in the Hippocampus in vitro. J Neurosci. 1999;19:RC18. 
79. Takahashi T, Kimoto T, Tanabe N, Hattori TA, Yasumatsu N, Kawato S. Corticosterone acutely prolonged N-methyl-d-aspartate receptor-mediated Ca2+ elevation in cultured rat hippocampal neurons. J Neurochem. 2010;83: 1441-51.

80. Liu X, Chen J, Guan T, Yao H, Zhang W, Guan Z, Wang Y. miRNAs and target genes in the blood as biomarkers for the early diagnosis of Parkinson's disease. BMC Syst Biol. 2019;13:10.

81. Wang X, Zhu Y, Wang S, Wang Z, Sun H, He Y, Yao W. Effects of eplerenone on cerebral aldosterone levels and brain lesions in spontaneously hypertensive rats. Clin Exp Hypertens. 2020;42:531-8.

82. Mascolo A, Sessa M, Scavone C, Angelis AD, Capuano A. New and old roles of the peripheral and brain renin-angiotensin-aldosterone system (RAAS): focus on cardiovascular and neurological diseases. Int J Cardiol. 2016;227:734.

\section{Publisher's Note}

Springer Nature remains neutral with regard to jurisdictional claims in published maps and institutional affiliations.

Ready to submit your research? Choose BMC and benefit from:

- fast, convenient online submission

- thorough peer review by experienced researchers in your field

- rapid publication on acceptance

- support for research data, including large and complex data types

- gold Open Access which fosters wider collaboration and increased citations

- maximum visibility for your research: over $100 \mathrm{M}$ website views per year

At $\mathrm{BMC}$, research is always in progress.

Learn more biomedcentral.com/submissions 